\title{
Influence of Management Competency on Performance of Family Planning Programs in Kuresoi North Sub-County, Nakuru County, Kenya
}

\author{
Jenifer Wothaya Wambugu, Dorothy Ndunge Kyalo, John Mbugua, and Regina Mutave
}

\begin{abstract}
This study investigated management competency influence on performance of family planning program in Kuresoi North Sub-County, Nakuru County, Kenya. The study was founded on the theory of constraint and adopted a pragmatism paradigm. It applied descriptive research method using mixed method approach to investigate the influence of management competency on performance of family planning program in Kuresoi North Sub-County, Nakuru County, Kenya. The target population in the study was women of reproductive age in 33,482 households distributed in the four Wards of Kuresoi North SubCounty and 19 health management leaders in-charge of all government health facilities offering family planning services in the Sub-County. A sample size of 400 of women of reproductive age and 19 nursing officers in-charge of government health facilities was utilized. The data collection tools used were questionnaire, interview guide, and observation checklist for women of reproductive age and the interview guide for the health facility managers. The research study adopted stratified sampling method. Qualitative data was analyzed according to themes and patterns and then summarizing the data and linking it to objectives and hypothesis. The study findings indicate that there is moderate management efficiency, exhibited pertaining to family planning in the dispensaries and health centers located in Kuresoi North Sub-County. Further findings exhibited that there was a significant and strong positive individual correlation between management competency and performance of family planning program in Kuresoi North Sub-County. Finally, the study findings also exhibited that there is a significant positive relationship between management competency and performance of family planning program in Kuresoi North Sub-County and it predicted the performance of family planning programmes. in Kuresoi North Sub-County.
\end{abstract}

Index Terms-management competency, family planning program, reproductive age.

\section{INTRODUCTION}

Family planning programs make a key starring role in prevention of maternal and infant mortality. Despite the benefits associated with family planning, many women continue to encounter unattained family planning needs leading to unwanted pregnancies and compromised health for women and children. The World Health Organization (WHO) in a 2018 report indicated that globally, two hundred and fourteen million females of procreative age from underdeveloped nations would like to use a family planning method for child spacing but continue to face challenges and barriers to accessibility.
A quality family planning service should ensure adherence, persistent, and positive referral of individual users as well as contribute to the overall improvement of health indicators especially maternal as well as neonatal health [1].

Efficiency is a performance of family planning program dimension which entails offering health care amenities in a technique that it is able to maximize the limited resource use and avoiding wastage. Accessibility is yet another performance of family planning program aspect, which comprises of offering health services that are opportune, physically reasonable, and offered in an atmosphere where knowledge, capacities and resources are suitable to health necessity and client counseling. Another performance of family planning program dimension identified was patientcentered which entails offering health care services, which considers individual or client predilections and goals, and the cultural setup of their societies. Another aspect is unbiassed, which is providing health care services that do not discriminate a person due to individual features like sex, race, culture, topographical setting, or financial standing. In addition, safety was considered as a performance of family planning program aspect. It entails providing health care services, which lessens jeopardies and damage to service consumers.

Achievement in programs related to family planning has been associated with key benefits to countries and the population. The major benefits as described by the WHO 2018 report are averting pregnancy-linked health dangers in women, dropping new-born mortality, supporting to avert HIV/AIDS, empowering individuals and improving education, as well as dropping adolescent pregnancies and decelerating population advance. Performance of programs related to contraceptives remains significant in attaining the United Nations Sustainable Development Goals. USAID through knowledge for health program revealed that there exists a gap in implementation of family planning worldwide.

Kenya made a great milestone in addressing quality in health by launching Kenya Quality Model of Health $(\mathrm{KQMH})$ care by [2], which provided a conceptual framework for offering holistic and scientific services through addressing a variety of structural quality matters with the key purpose of delivering health effects that are positive. 
Management competency encompasses the overall effectiveness of a program and it entails offering health care that is in line with the evidence generated through studies and results in better-quality health results for persons and societies. Further management competency indicates that the program should be based on requirements such as commodity supply.

Thus, management competency in family planning programs comprises of providing family planning services that are in line with an indication base and outcomes in better-quality family planning results for persons and societies and are aligned to the need. There are several components of management competency dimension. These include leadership, accurate report documentation, and adherence to guidelines.

Management competency in family planning involves combining many strategies that can be effective in a program. This is in convergence with a study by [3] that described offering superior family planning facilities in disasteraffected locations, described strategies that could be borrowed from successful countries on family planning programs. Some of the strategies that had worked in other program included capability-founded training, structured support supervision, modest quality enhancement tools, communal mobilization, as well as execution of quality development and clinical management through the government health structure and building nationwide capacity. Management competency therefore requires managers to view the program in a holistic way.

Despite the launch of the KQMH care document, there is no study conducted on the implementation of KQMH and the influence on performance of family planning program in Nakuru County. Family planning services are very critical services in preventive health especially at primary health care level. This study has utilized the healthcare quality dimensions of management competency in relation to performance of family planning program in Nakuru County, focusing on Kuresoi North Sub-County.

The remaining part of this paper is arranged as follows. Section two discusses about literature reviews. Section three deals with data and research methodology. The last two sections focus on empirical analysis and conclusions respectively.

\section{LITERATURE REVIEW}

Management competency in family planning comprises of delivering of family planning amenities that are devoted to an indication gained through research and outcomes in better-quality health results for persons and societies, built on requirement. Access to quality health care is a human right, which calls for leadership to adhere to the legal and moral obligation in providing the care. Leadership is of paramount importance in achieving total quality of care. KQMH describes leadership as a process that provides guidance and motivation to improve quality care hence the role of leadership is key in achieving the total quality of care.

The model furthers described leadership in total quality management as that of maintaining a conducive environment that enables staff to be part of the process towards achieving the organization's purposes or goals, validate obligation to the group or organization, and support employees to overcome ordinary confrontation to transformation and to persuade staff that quality is vital. Leadership role should be clear, precise and well defined on how to objectively meet the threshold of guiding the total quality management.

A good performing organization usually has a culture that helps to maintain standard all through, organization culture. In health care, the culture needs to be nurtured, and health care providers mentored on by the organization leadership. This would ensure quality improvement, safe and compassion health care, which has remained a key challenge in health sector. There are numerous researches steered on the position of leadership in enhancing a culture of continual improvement. Leaders have a great role in sustaining a culture as revealed by [4] in a research on leadership development in England.

The study indicated that many institutions were encountering a challenge of lack of a culture of quality delivery and continuous improvement making many organizations to stagnate in quality of care especially in health care. The study also added that it was the role of management to enhance a workplace with refining extreme quality, harmless, and empathetic healthcare services. The dominant impact of leadership in any institution is very important. Leadership therefore has a great role to ensure adherence to the organization values, strategies, and vision. The study however failed to provide a guideline on how leaders can provide the mentorship.

A study carried out by [5] had convergence in the responsibilities of leadership in building and maintaining organization principles and sought to investigate organization philosophy in the English National Health Service (NHS). The study identified some key components, which were of paramount importance towards sustaining values that ensure excellence and compassionate care for clients. The study outlined some elements of excellence. These include inspirational dreams operationalized at all levels of leadership in health facilities and community primary care. Leaders aligned objectives for all players with inclusion of stakeholders, branches, and specific staff, which are helpful and empowering individual's administration. The objectives were; elevated staff assignation level, leaders engrossed to affirming education especially eLearning, invention to get new way of addressing quality and quality enhancement as a culture by all workers, as well as actual teamwork. The study did not consider the working environment as part of leadership role in ensuring quality.

Leadership style in health care has been associated with saving of life's, which was also revealed in a study by [6] on patients' outcome and leadership style. The discoveries suggested that there is a correlation between nursing leadership style and the numbers of mortality in the hospital as well as medication errors, while the leadership style plays a key part in health upkeep. The study however failed to establish the specific style of leadership in relation to various context of giving medical care. 
In divergence with the results, another extensive assessment of therapeutic management model carried out by [7] established that various leadership styles vary according to the medical or clinical site. Leadership style may therefore not be used uniformly in all health sector setups. Selection of the right manager with the right skills to manage a health facility is very important in determining the overall performance in health care, patient satisfaction, and morbidity rates. The finding further indicated that leadership required support from the political angle in order to practice the strategies that work for an institution as well for the users of the health facility. The leaders therefore need to have a background on the issues affecting institutions and hence advocacy remains a key tool in sensitizing leaders.

This is in convergence with a study on National Health Service hospital trusts by [8], which established that the health facilities with clinicians governing the boards had greater performance. The study however failed to establish whether the clinicians governing the health facilities had undertaken a management course since managerial skill are important in health sector management.

Job satisfaction can be derived from the type of leadership. In a study by [9], on leadership and work life, found that reliable management is associated with job satisfaction and employees portraying characteristics of leadership like trustworthiness, unselfishness, compassion, justice, responsibility, and hopefulness and this legitimacy indicates steadiness with morals of offering excellence and empathetic client care. The intellectual capacity of leaders determines how well leaders take up their roles in health care. The findings were also noted by [10] in a study that outlined that a front-runner within the health segment must understand the factors behind good performance. Some of the factors include; understanding on performance matters, value aspect of care, as well as formation of performance improvements require teamwork. The study brought about a new concept of value for service which is key for leaders to evaluate whether the clients get value for their cost in relation of the services they receive in health.

Health care workers plays a crucial part in advocating and promoting for any service. Providing knowledge regarding family planning can improve uptake of family planning. Health service providers need to give well-structured information to clients during visits on health facilities since lack of information affects a woman's ability to make an informed decision on uptake of family planning [11]. Programs need to identify the strength and level of trust on health care providers and build on that trust to advocate for family planning.

\section{A. Theoretical Review}

The theory that the study was grounded on was the theory of constraint. This is because constraints are known to determine the implementation and performance of any system. In order to focus on excellence in management, together theoretical and functional aspects must be considered as described by [12]. In their recommendation, handling excellence should be the guide in the execution of any project. They further described a constraint as everything that averts a system from attaining excellence performance reasonable to its goal.

The Theory of Constraints was established by Dr Eli Goldratt and pronounced in his narratives, attentions on accomplishing breakthroughs in performance in big multifaceted settings, subjugated by high ambiguity. He further described bottleneck and contract. Bottleneck was defined as any resource whose dimensions is less than the mandate placed upon it: Bottlenecks come and go. A constraint limits the whole organizations performance over a stretched period. Each chain up maintains the lowest link, the intensity of such a chain up is established by the lowest link.

In a study by [13] on measure contract found that what is measured as a constraint in project management can be patented in to four. These are as party-political constraints which include factors such as indistinct dreams, undertaking and scope of projects, mechanical constraints which includes individual strengths such as competences, skills, prevailing structure and normal conditions like geology, location and climate, communal constraints which includes codes of behaviour, organizational protocols, individual relationships, acknowledged or predictable behaviour's, and managerial constraints like budgets, project plans, scope, inscribed contractual arrangements, among others.

Theory of Constraints contests administrators to volte-face some of their essential assumptions about how to accomplish the goals of their organizations, about what they contemplate creative actions, and about the actual determination of cost administration. In another research finding, by [14], they described theory of Constraints as an organization thinking whereby companies can be deemed as a mutually dependent sequence of procedures instead of an impartial enterprise component.

Theory of constraint describes five steps which includes; Documentation of the scheme's constraint, verdict on how to exploit the organisation's constraint, subordination of all other issues to the above decision, advancement of the system's constraint and lastly if in the preceding steps a constraint has been cracked, one should start all over again from step one.

The theory applies to the current study to the extent that in order to measure management competency, an organization needs to identify the five steps in the theory of constraint to address any constraint that can hinder management competency.

The study also will also apply the Donabedian's model for measuring quality care. [15] developed three mechanisms for measuring the quality of care. These mechanisms are structure, process, and outcomes. Measurement for enhancement has an added component which is referred to as balancing measures. [15] thought that structure usually impact on processes while processes in turn affect the outcome of any project. The three; structural, process, and outcome, comprises quality. Measure of quality should therefore assess the three components.

Outcome measures: These replicate the effect on the client and validate the result of the development of effort and if it has lastly accomplished the set objectives. An example of 
result measures is upsurge in the couple year of protection, increase in acceptance of contraceptives procedures.

Process measures: These replicate the way a systems and procedures lab to bring the anticipated results. An example of process measures includes the client waiting time to receive a contraceptive service. Structure measures: These echo the characteristics of the health care provider for instance staff to client proportions and functioning spells of the service. They can also be referred to as input measures.

Balancing measures: This mirror unintentional and/or wider consequence of the transformation that can be optimistic or undesirable. It is about identifying these and endeavouring to measure them and or reduce their effect if necessary. An example of a balancing measure would be monitoring rates use of family planning after initiating a community advocacy program for family planning.

Utilization of quality assessment in sectors such as health is seen to have possibility of advancing care quality as well as maximizing the capability and prevent ordinary drawbacks. In addition, they indicated the need to utilize the past practice in order to come up with a proper tool to assess quality at all levels of health care. In this study, management competency is viewed as a key to addressing challenges in the health sector, particularly family planning programs, and how to improve the programs [16].

\section{DATA AND RESEARCH METHODOLOGY}

This section profiles the research procedures that were applied to address the research objective. These included research model, research strategy, target populace, sample size, and sampling methods, research tools, data gathering methods, data analysis systems, ethical attention and operational description of variables. It also describes the procedures undertaken to guarantee the validity as well as the reliability of the investigation instruments. The section equally includes the ethical concerns. The section concludes with the description of variables.

\section{A. Research Paradigm}

This study was steered by pragmatisms paradigm since the study integrated quantitative and qualitative research strategies by use of mixed technique of data collection and examination. The fundamental hypothesis of the mixed study methodology is that blending numerical and non-numerical techniques offers a comprehensive knowledge of the investigation problem compared to applying only single kind of techniques as described by [17] and [18]. This is in concurrence with discoveries from a study by [19] who described dual case studies that utilized mixed study approaches and revealed that use of mixed study delivered both quantitative as well as qualitative perceptive which resulted to superlative data elucidation as well as the superlative understanding of the investigation phenomena.

Mixed approaches research provided additional thoughtful of the multifaceted phenomena that was likely to otherwise not be available through using single method only as described by [20]. Pragmatism paradigm was carefully chosen as the most appropriate for this survey since the survey aimed to examine the influence of healthcare quality dimensions and client characteristics on performance of family planning program in Kuresoi North Sub-County, Nakuru County, Kenya.

The choice of the paradigm was to accommodate the diverse approaches of data gathering as indicated by [21] that pragmatism is predominantly suitable for mixed methods. In addition, pragmatism permitted the investigator to be free of psychological and hands-on constrictions executed by the "involuntary optimal contradiction amid post positivism and constructivism as described by [20]. It also enabled the investigator to be independent hence not obliged to a certain research method or technique as stated by [20].

\section{B. Research Design}

The study utilized the descriptive survey using mixed techniques research design. Mixed method is a procedure for steering research that encompasses gathering, scrutinizing and assimilating quantifiable statistics such as surveys and qualitative statistics such as interviews and observation guide as described by [20]. The choice of mixed method was to offer a better consideration of the research problem. The method was selected considering that there had not been a study in Kuresoi North Sub-County testing the effect of management competence on any health program especially family planning. It was of paramount important to therefore use a method that would enable the investigator to continuously review the research question from diverse angles and elucidate unforeseen discoveries and/or probable contradictions.

Use of mixed method approach gave advantage to the study as described by [23] whereby, through use of the approach, the investigator was able to examine uniformity of outcomes obtained by various tools which included; household survey, interview guide, and observation checklist. It also offered data complementation by using both numerical and nonnumerical statistics to evaluate overlapping but distinctive aspects of the research phenomenon.

\section{Target Population}

The target population in this study was 33,482 households in Kuresoi North Sub-County, Nakuru County. This number of households in Kuresoi North Sub-County was drawn from the Kenya health information system. The study also targeted the 19 nursing officers in-charge of all government health care facilities offering contraceptive services in Kuresoi North Sub-County.

This target population was useful in providing the required data in the topic under investigation, which was management competency and performance of family planning program in Kuresoi North Sub-County, Nakuru County, Kenya. The target population of 33,482 households was guided by the households mapped in Kuresoi North Sub-County as per the 2009 Census and 4\% estimated annual population growth as tabulated in the Kenya health information system 2019. The women of procreative age in Kuresoi North Sub-County was equally guided by 2019 Population Estimates revised for Kuresoi North Sub-County as populated in the Kenya Health Information system developed in 2019. This estimate of 
population and households is usually calculated guided by the population as per the census and the annual growth rate per Sub-County in relation to the proportion of each age cohort. Kuresoi North Sub-County has an estimated growth rate of $4 \%$ per year.

The health care facility in-charges comprised of all 19 officers of government health care facilities in Kuresoi North Sub-County. The women of procreative age were reached at the household level by trained community health volunteers. The estimated number of households in Kuresoi Sub-County were 33,482 which accounts $19.2 \%$ of the total population distributed in the four wards of the Sub-County. The inclusion of the health facility managers in this study was to enhance an understanding on management competency and performance of the family planning program.

The inclusion criterion for women of procreative age was the women within the procreative age cohort in a household that were eligible for collecting data from. The exclusion criteria were women of non-procreative age as per the operational definition irrespective of utilizing family planning services. The inclusion criteria for health care facility in-charges was only the nursing officers in-charge of all the government health facilities in Kuresoi North offering family planning services while the exclusion criteria were private health care facility nursing officers in-charge irrespective of offering family planning.

\section{Sample and Sampling Procedures}

Sample scope that was utilized for the survey was taken by applying Slovin's method designated by the populace size and the standard allowance error of 0.05 as specified in the equation below.

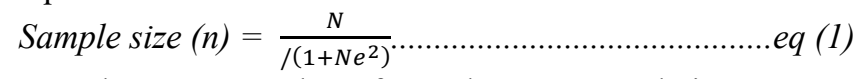

Where; $\mathrm{n}=$ number of samples, $\mathrm{N}=$ population scope, $\mathrm{e}$ $=$ margin of index or error margin. The error margin employed in the study was $5 \%$ as the confidence interval utilized for social sciences is $95 \%$ and the consequent error margin is $5 \%$. Hence, the sample size obtained for the study was 400 respondents.

The sample was allocated in the four wards using the fisheries method for stratified random sampling procedure. The purpose of this method was to maximize sample survey precision from the calculated sample size. This is enumerated in Table I.

The population, which was being investigated, was homogenous, the study adopted stratified random sampling technique. The definition is in convergence with description by [24] who defined stratified random sampling as a process by which populace is segregated into subcategories known as "strata". Out of which every stratum, the same random sampling is applied in choosing for each-stratum sample. The overall each-stratum samples are thereafter joined to come up with the stratified random sample. It encompassed dividing the populace into standardised subdivisions and then getting a simple random sample in each subcategory.

\begin{tabular}{ll}
$\mathbf{N}$ & TABLE I: SAMPLING FRAME \\
\hline$M_{\mathrm{i}}=\sum_{j=1}^{\text {Number of stratum (SU1) in the }}$ \\
population
\end{tabular}

\section{E. Data Collection Procedures}

The research assistants were guided by the Ward administrator, community health volunteer, the area chief, area assistant chief, and the village elder to identify a central point in each Ward, which was to be the initial point for sampling the households in each ward. The ward was further dived into four routes which informed the starting point. Each direction or route had equal number of questionnaires per the ward. To determine the starting point of data collection, the research assistant rolled a pen on the ground. The direction the sharp edge pointed was the initial route used to commence data collection. The research assistants further visited the first household and then skipped two households and visited every third household in the selected direction.

The research assistants ensured that they always started from the central starting point in the four routes selected. Upon getting to the household, the research assistants introduced her/himself, sought to know if the household had a woman of reproductive age, and if present, and sought permission from the household head or equivalent to administer the household questionnaire with assurance of confidentiality. For the households that did not have a 
woman of childbearing age, the research assistant moved to next household without skipping then continued with the pattern of skipping two households.

This study utilized a household questionnaire, key informant interview guide, and observation checklist for data collection. The survey tool for women of procreative age in the designate wards was a questionnaire. The key informant interview guide was utilized among the in-charges of health care facilities. Observation checklist was utilized to assess the nineteen health care facilities in Kuresoi North SubCounty. The choice of these instruments was directed by the type of data to be gathered, the dimensions and distribution of the population and also the goal of the research. The use of questionnaire in this study was carefully chosen since it allowed the questioner to gather a more deep and comprehensive information since the interviewer could selfcontrol the procedure henceforth searching additional by adding questions that aided to add additional information dissimilar in an observation method

The questionnaire and interview guide were tested in Molo Sub-County, Nakuru County, Kenya. The pilot test targeted 15 women of procreative age. The choice of Molo SubCounty in Nakuru county was because it was not a study site. The two health facilities were randomly selected in Molo sub-county. The process of piloting was aimed at identifying whether women of reproductive age would understand the interrogations and instructions as well as whether the substance of questions was the similar for all respondents. For the household questionnaire, which had closed ended questions, piloting helped to check the sufficiency of response in the categories that were available. The responses from the participants was also expected to reveal presence of any inconsistencies in the questions within the questionnaire and ability of respondent to respond to all questions. The pilot study gave proper guidance on the tools. The tool collected the data which was intended hence they was no need of revising the tools.

\section{F. Research Instruments Validity and Reliability}

Inferences around the uses of the research apparatuses or instruments was validated. The validation was carried out to ensure that the research instruments had a suitable inference relevant to the purpose of the study and that the research instrument had a meaningful inference hence giving meaningful information using the instrument. The research instrument was tested to divulge the three categories of validity, which were; content-interrelated indication of validity, the criterion-interrelated indication of validity and the construct interrelated indication of validity. Utilization of experts in the area of reproductive health was done to help in assessing the subject matter validity. The tool was reviewed by the county health management team for any error. A small team that comprised of County Director of Strategic Planning, County Director of Quality Assurance and County reproductive health coordinator were tasked to review and give feedback on content. The review was followed by a presentation to the county health management team and later permit to collect data was given. The researcher put into consideration sentiments of raised by the Nakuru County Health Management Committee.

To ensure reliability of the research tools, they were exposed to testing to guarantee that the features of steadiness and equivalency were certain. The method used to measure reliability of the study tools was test re-test method. The interview guide was tested in two health facilities in Molo Sub-County targeting health facility in-charges while the questionnaire for the women of procreative age was tested in Kuresoi North Sub-County interviewing 20 women of procreative age at house level. The women of procreative age were visited in their respective households, explained to about the survey and upon giving consent, they were interviewed. They were given an appointment of one month when the researcher returned to the same households and interviewed the same women using the same questionnaire. The two-health facilities in-charge were interviewed utilizing the interview manual and the given an appointment of one month after which the same interview manual was utilized to interview them. The results of the two sets of were calculated for correlation using SPSS.

\section{G. Data Analysis Techniques}

This survey produced both quantitative and qualitative data to describe the influence of management competency on performance of family planning program. The filled questionnaires were obtained, coded, and edited for comprehensiveness and steadiness. The data was examined by applying descriptive and inferential statistics using the Statistical Package for Social Science (SPSS). This was utilized to give both descriptive and inferential statistics which enhanced examination of the hypothesis at the significance level of 0.05 and the confidence interval of $95 \%$.

Qualitative data was analyzed from the in-depth interviews and observation checklist and involved analyzing transcripts and identifying themes within that data. This also involved putting together themes that were alike from the text thus qualitative data was analyzed by checking data, developing codes, identifying themes and patterns, and then summarizing the data and linking them to objectives and hypothesis.

A simple linear regression analysis was carried out to analyze the influence of management competency on the performance of family planning program. The hypothesis was also analyzed using Pearson's Product Moment Correlation for interpretation of results. Correlation analysis was key in order to determine the association between the independent and dependent variables. Finally, the data was analyzed descriptive statistics for the quantitative analysis of data. The data was then be presented using frequency distribution tables for easier understanding.

\section{EMPIRICAL ANALYSIS}

The empirical analysis section contains four parts which include, descriptive statistics, content analysis, observation 
chart, correlation analysis, simple linear regression analysis, and discussion of empirical findings.

\section{A. Descriptive Statistics}

The study sought to determine the mean, standard error and standard deviation of management competency. This was analyzed using SPSS. There were ten questions distributed among the four indicators of the independent variable (management competency). The mean, standard error and standard deviation was analyzed as per as the results in the in Table II below.

TABLE II: MANAGEMENT COMPETENCY DESCRIPTIVE STATISTICS

\begin{tabular}{ll|lll}
\hline & N & Mean & & $\begin{array}{l}\text { Std. } \\
\text { Deviation }\end{array}$ \\
& Statistic & Statistic & $\begin{array}{l}\text { Std. } \\
\text { Error }\end{array}$ & Statistic \\
\hline $\begin{array}{l}\text { Management } \\
\text { Competency }\end{array}$ & 400 & 30.69 & .498 & 9.960 \\
\hline Valid N (listwise) & 400 & & & \\
\hline
\end{tabular}

The results exhibit a mean of 30.69 , standard error of 0.498 , and standard deviation of 9.960. The interpretation is that; about $68 \%$ of the sample population, with an assumption of normal distribution, at one standard deviation is between $(30.69-0.498)=30.192$ and $(30.69+0.498)=31.188$ which has an average of 30.69. This indicated that the sample population tended to be neutral on the management of the health facility they were seeking contraceptive services. The views of the sample population gave the same views with the total population which was between $30.69-9.960=20.73$ and $30.69+9.960=40.65$ with average of 30.69 .

The study also sought to measure the mean and standard deviation of performance of family planning program. Table III shows the results.

TABLE III: PERFORMANCE OF FAMILY PLANNING PROGRAM

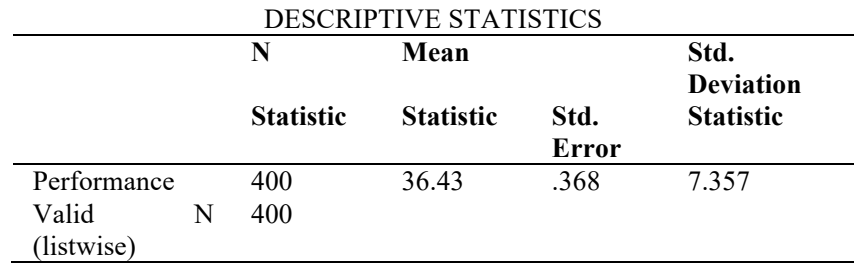

The results display a mean of 36.43 , SE of 0.368 and SD of 7.357. This implies that $68 \%$ of the sample population at one standard deviation is between $(36.43-0.368)=36.0621$ and $(36.43+0.368)=36.798$, which has an average of 36.43 . This indicated that the sample population tended to agree on the performance of family planning program. The views of the sample population gave the same views with the total population, which was between $36.43-7.357=29.073$ and $36.43+7.357=43.787$ with average of 36.43 .

\section{B. Content Analysis}

Content analysis was conducted on questions asked in the interview guide pertaining to management competency. The findings are enumerated below. Nursing officers in-charge of nineteen government health facilities in Kuresoi North were interviewed to get their opinion on management and the general leadership. On the question on how the county leadership ensured that the staff were updated in their skills,
$100 \%$ of the nursing officers were in agreement that the county and the sub-country leadership was able to ensure that they remained updated with all the relevant skill. They cited that leadership enhanced skills transfers through integrated outreaches whereby a senior officer carries out mentorship especially on long-term family planning procedures. Other avenues for skills transfer that that the county was using was using continuous medical education sessions, on the job training, through the job aids and information charts, through review meeting and one on one sessions during supportive supervision.

The question on evidence that staff are regularly updated was asked. Nursing officers had different ways that is used to measure update of health care workers. $50 \%$ of the nursing officers stated that the measure of updates was through the minutes of the records kept by the facility after every update. $30 \%$ felt that the best measure was through assessing quality of care and improvement of services, which could be checked through Kenya Health Information System (KHIS). The KHIS would show an upsurge of some services. 20\% indicated that improvement of skills could be measured through supportive supervision where skills are tested, and recap of the skills is done.

The nursing officers were also asked on other ways that the county and sub-county leadership managed to ensure that nursing officers were up to date with skills and knowledge on family planning. They indicated that social media forum like a WhatsApp group were utilized where some urgent information is given to members awaiting a formal discussion. Through the group, videos are shared, and other job aids that facilitated the health care workers to learn more. The management can explore the idea of eLearning through mobile phones since all the nursing officers own a smart phone and are connected to others through what up group.

On the question of how the health care workers client documentation was asked. The health care workers stated that they were documented family planning services in the family planning registers. They said that the hospitals lacked the family planning card for mothers and hence use exercise books that clients buy. They also indicated that they were not able to trace the women who missed family planning appointments. This was also the case with the women of reproductive age who indicated a gap in documentation during the household survey that showed that women tended to disagree on the few management issues especially on documentation.

\section{Observation Chart}

The study investigated whether the 19 dispensaries and health centers in Kuresoi North Sub-County correctly filed family planning reports. Six parameters of filing the reports were chosen. The findings are presented in Fig 1. 


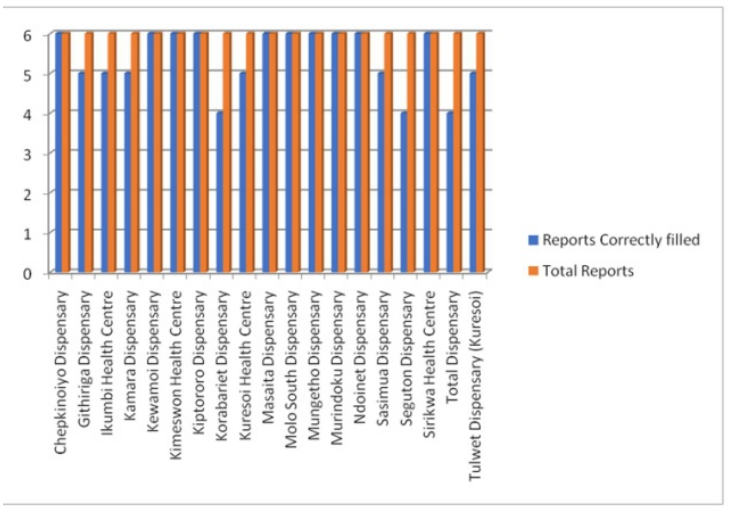

Fig. 1. Filing of family planning reports.

The findings showcased that 10 of the dispensaries and health centers observed all the six parameters in the correct filing of the family planning reports. Three dispensaries observed the lowest number of parameters, which were 4 parameters.

The study further investigated whether the 19 dispensaries and health centers implemented tools and skills for ordering of contraceptives. One tool and one skill were chosen to asses efficient ordering of contraceptives, translating to two parameters. The results are displayed in Fig 2.

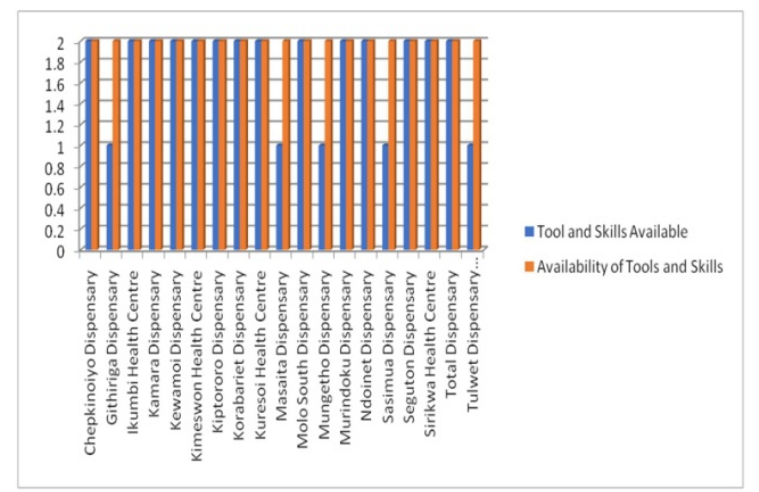

Fig. 2. Tools and skills for ordering of contraceptives.

The findings highlight that 14 of the dispensaries and health centers attained the 2 parameters in ordering of contraceptives. The balance attained at least one of the parameters.

\section{Correlation Analysis}

The study sought to establish the influence of management competency on performance of family planning program in Kuresoi North Sub-County, Nakuru County, Kenya. Management competency as an independent variable was guided by four indicators which were; leadership, health care providers skills and level of training, adherence to guidelines, and report and documentation. Table IV shows the correlation.

The study identified a strong positive correlation of 0.624 (62.4\%) between management competency and performance of family planning program in Kuresoi North Sub-County and a significance level of 0.00 which is less than the significance level of 0.05 . Thus, there is a significance association between management competency and performance of family planning program.
TABLE IV: CORRELATIONS ANALYSIS

\begin{tabular}{|c|c|c|c|}
\hline & & $\begin{array}{l}\text { Management } \\
\text { Competency }\end{array}$ & Performance \\
\hline Management & Pearson & 1 & $.624^{* *}$ \\
\hline \multirow[t]{3}{*}{ Competency } & Correlation & & \\
\hline & Sig. (2-tailed) & & .000 \\
\hline & $\mathrm{N}$ & 400 & 400 \\
\hline \multirow[t]{3}{*}{ Performance } & $\begin{array}{l}\text { Pearson } \\
\text { Correlation }\end{array}$ & $.624^{* *}$ & 1 \\
\hline & Sig. (2-tailed) & .000 & \\
\hline & $\mathrm{N}$ & 400 & 400 \\
\hline
\end{tabular}

\section{E. Simple Linear Regression Analysis}

The study sought to test the fitness of the model on relationships between management competency and performance of family planning programs. The study also sought to test hypothesis that there is no significant relationship between management competency and performance of family planning program. Finally, the study sought to measure the strength of the relationship between management competency and performance of family planning program. Tables 4.12 shows the results of the model.

TABLE V: SIMPLE LINEAR REGRESSION ANALYSIS

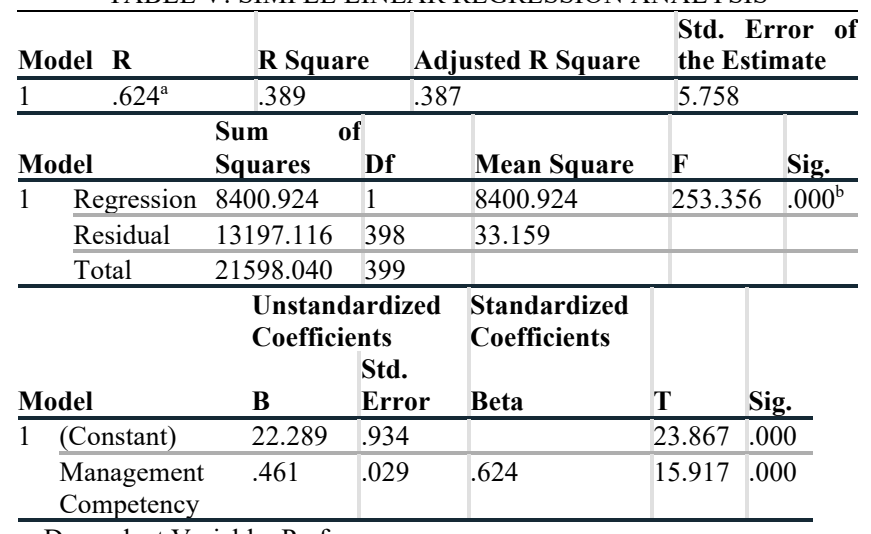

a. Dependent Variable: Performance

b. Predictors: (Constant), Management Competency

The results showed that R Square value was 0.389 . Thus, management competency predicts $38.9 \%$ of performance of the family planning program in Kuresoi North Sub-County, Nakuru County, Kenya.

The result also indicated that significance value obtained in the analysis of variance is 0.00 , which is less than the critical of 0.05 . Thus, the null hypothesis that management competency does not predict performance of family planning program is rejected. Hence, the model of management competency predicting performance is therefore fit.

The result indicated that significance value obtained from the model coefficients is 0.00 which is less than the critical value of 0.05 . Thus, management competency significantly affects performance, the null hypothesis that management competency does not significantly affect performance of family planning program is rejected. The following model is thus developed;

$$
Y=22.89+0.461 X
$$

This implies that when one improves management competency by one unit, there is an increase in performance of family planning program by 0.461 units.

\section{F. Discussion of Findings}


The study findings displayed that there was a significant and strong positive correlation between management competency and performance of family planning program in Kuresoi North Sub-County. The study findings also exhibited that there is a significant positive relationship between management competency and performance of family planning program in Kuresoi North Sub-County. The study further exhibited that management competency predicted and described to a large extent the performance of family planning.

The study findings are in tandem with the conclusions of [2] that leadership is of paramount importance in achieving total quality of care. The report stipulated that leadership is a process that provides guidance and motivation to improve quality care hence the role of leadership is key in achieving the total quality of care. The model developed in the report further described the main objective of leadership in total quality management as that of maintaining a conducive environment that enables staff to be part of the process towards achieving the organization's purposes or goals, validate obligation to the group or organization and support to overcome employees ordinary confrontation to transformation and to persuade staff that quality is vital and that leadership role should be clear, precise and well defined on how to objectively meet the threshold of guiding the total quality management.

The study findings are also congruent to findings of the study conducted by [5], which established that a good performing organization usually has a culture that helps to maintain standard all through, organization culture and in health care, the culture needs to be nurtured, and health care providers mentored on by the organization leadership. The study opined that this would ensure quality improvement, safe and compassion health care which has remained a key challenge in health sector.

The study findings are parallel to findings of a report of English National Health Service (NHS), carried out by [3] which sought to investigate organization philosophy in the institution. The study identified some key components which were of paramount importance towards sustaining values that ensure excellence and compassionate care for clients which included; inspirational dreams operationalized at all levels of leadership in health facilities and community primary care, leaders aligned objectives for all players with inclusion of stakeholders, branches and specific staff, helpful and empowering individual's administration, elevated staff assignation level, leaders engrossed to affirming education especially eLearning, and invention to get new way of addressing quality and quality enhancement as a culture by all workers, as well as actual teamwork.

The study findings are in agreement with a study conducted by [6] on patients' outcome and leadership style which outlined that leadership style in health care has been associated with saving of lives and suggested that there is a correlation between nursing leadership style and the numbers of mortality in the hospital as well as medication errors.

The study findings are in tandem with those of a study carried out by [7], which was another extensive assessment of therapeutic management model that established that various leadership styles vary according to the medical or clinical site. Leadership style may therefore not be used uniformly in all health sector setups. Selection of the right manager with the right skills to manage a health facility is very important in determining the overall performance in health care, patient satisfaction and morbidity rates. The finding further indicated that leadership required support from the political angle in order to practice the strategies that work for an institution as well for the users of the health facility. The leaders therefore need to have a background on the issues affecting institutions and hence advocacy remains a key tool in sensitizing leaders.

The study findings are in convergence with a study on National Health Service hospital trusts by [8], which established that the health facilities with clinicians governing the boards had greater performance. The study findings are also in agreement with a study by [9] on leadership and work life which found that reliable management is associated with job satisfaction and employees portraying characteristics of leadership like trustworthiness, unselfishness, compassion, justice, responsibility, and hopefulness legitimacy indicates steadiness with morals of offering excellence and empathetic client care and that the intellectual capacity of leaders determines how well leaders take up their roles in health care.

The current study findings are congruent to the study done by [10], which noted that a front-runner within the health segment must understand the factors behind good as performance. Some of the factors outlined included understanding on performance matters, value aspect of care as well as formation of performance improvements require teamwork. The study also brought about a new concept of value for service which is key for leaders to evaluate whether the clients get value for their cost in relation of the services they receive in health.

\section{CONCLUSIONS}

The study concluded that there was a significant and strong positive correlation between management competency and performance of family planning program in Kuresoi North Sub-County.

The study also concluded that there is a significant positive relationship between management competency and performance of family planning program in Kuresoi North Sub-County. The study finally concluded that management competency predicted and described to a large extent the performance of family planning.

Thus, recommendations can be made to the health regulatory authorities and health facilities to increase their management competencies in order to increase the performance of family planning programs. In order to increase management competency the health care providers should; offer quality health care, improve leadership of the health facility, document all findings and properly file all records, offer services in the shortest time possible, have a clearly written charter indicating services offered and cost, adheres to the time indicated for the service, have a suggestion box to obtain customer feedback, and address complaint raised by clients 
The study conclusions are in tandem with the conclusions of [2] that leadership is of paramount importance in achieving total quality of care. The report stipulated that leadership is a process that provides guidance and motivation to improve quality care hence the role of leadership is key in achieving the total quality of care. The model developed in the report further described the main objective of leadership in total quality management as that of maintaining a conducive environment that enables staff to be part of the process towards achieving the organization's purposes or goals, validate obligation to the group or organization and support to overcome employees ordinary confrontation to transformation and to persuade staff that quality is vital and that leadership role should be clear, precise and well defined on how to objectively meet the threshold of guiding the total quality management.

The study conclusions are also congruent to findings of the study conducted by [5], which established that a good performing organization usually has a culture that helps to maintain standard all through, organization culture and in health care, the culture needs to be nurtured, and health care providers mentored on by the organization leadership. The study opined that this would ensure quality improvement, safe and compassion health care which has remained a key challenge in health sector.

\section{REFERENCES}

[1] [S. Cohen and W. Yu. Rockville, "The Concentration and persistence in the level of health expenditures over time: Estimates for the U.S. population 2009-2010," MD: Agency for Healthcare Research and Quality; Nov, 2012.

[2] Implementation Guideline for Kenya Quality Model of Health (KQMH) Care, Ministry of Medical services and Ministry of Public Health and sanitation, 2012.

[3] D. M. Woods, R. Baker, and K. Charles, "Culture and behaviour in the English National Health Service: overview of lessons from a large multimethod study," BMJ Qual Saf, vol, 23, no 2, pp 106-15, 2014.

[4] D. W. Curry, J. Rattan, J. J. Nzau, and K. Giri, "Delivering HighQuality Family Planning Services in Crisis-Affected Settings I: Program Implementation," Global Health: Science and Practice, vol 3, no 1, pp 14-24, 2015.

[5] M. West, K. Armit, L. Loewenthal, R. Eckert, T. West, A. Lee, (April, 2015). Leadership and leadership development in health care: The evidence base. Available from http:ororwww.kingsfund.org.ukorpublicationsorleadershipand-leadership-developmenthealth-care What are patients' knowledge, expectation and experience of radial extracorporeal shockwave therapy for the treatment of their tendinopathies? A qualitative study

[6] C. Wong, C. G. Cummings, and L. Ducharme, "The relationship between nursing leadership and patient outcomes: A systematic review update," J Nurs Manag, vol 21, no5, pp 709-24, 2013.

[7] H. Dickinson, C. Ham, and I. Snelling, "Are we there yet ? Models of medical leadership and their effectiveness, An exploratory study," Final report. NIHR Service Delivery and Organisation programme, 2013.

[8] G. Veronesi, I. Kirkpatrick, F. Vallascas, "Clinicians on the board: what difference does it make?," Soc Sci Med, vol 77. pp 147-55, (2013)

[9] C. A. Wong, and H. K. Laschinger, "Authentic leadership, performance, and job satisfaction: the mediating role of empowerment," J Adv Nurs, vol 69, no 4, pp 947-59, 2013.

[10] A. C. Arroliga, C. Huber, J. D. Myers, J. P. Dieckert, and D. Wesson, "Leadership in health care for the 21st century: Challenges and opportunities," Am J Med, vol 127, no 3, pp 246-249, 2014.

[11] T. C. Okech, N. W. Wawire, and T. K. Mburu, "Contraceptive use among women of reproductive age in Kenya's city slums"
International Journal of Business and Social Science, Vol. 2, No. 1, 2011.

[12] G. Saad, \& S. Siha, "Managing quality: critical links and a contingency model", International Journal of Operations and Production Management, Vol. 20 No. 10, pp. 1146-1164, 2000.

[13] S. Lombardo, and R. Kvålshaugen, (2014). Constraint-Shattering Practices and Creative Action in Organizations. https://doi.org/10.1177/0170840613517597.

[14] A. Sabbaghi, and G. Vaidyanathan, "SWOT Analysis and Theory of Constraint in Information Technology Projects," Information Systems Education Journal, vol 2, no 23, 2004.

[15] A. Donabedian, "Evaluating the quality of medical care," The Milbank Quarterly, vol 83, no 4, pp :691-729, 2005.

[16] V. S Raleigh, and C, Foot, Getting the Measure of Quality: Opportunities and Challenges, London: King's Fund, 2010.

[17] J. W. Creswell, Research Design: Qualitative, Quantitative, and Mixed Methods Approaches, 4th ed, 2014.

[18] J. F. Molina-Azorin, "Mixed methods research: An opportunity to improve our studies and our research skills," European Journal of Management and Business Economics, vol 25, pp 37-38, 2016.

[19] A. Mitchell, "A review of the mixed methods, pragmatism and abduction techniques," The Electronic Journal of Business Research Methods, vol 16, pp 103-116, 2018.

[20] J. W. Creswell, and V. L Plano-Clark, Designing and Conducting Mixed Methods Research,2nd ed., Thousand Oaks, CA: Sage Publications, Inc, 2018.

[21] D. L. Morgan, pragmatism as a paradigm for social research, Qualitative Inquiry, vol 20, no 8, pp 1045-1053, 2014.

[22] C. Robson, Real World Research, Oxford, UK: Blackwell, 1993.

[23] J. Morse, and L. Niehaus, Mixed Method Design: Principles and Procedures, Walnut Creek, CA: Left Coast Press, 2010.

[24] T. D. Nguyen, M. Shih, D. Srivastava, S. Tirthapura, and B. Xu, "Stratified Random Sampling from Streaming and Stored Data," Electrical and Computer Engineering Conference Papers, Posters and Presentations, 2019. 


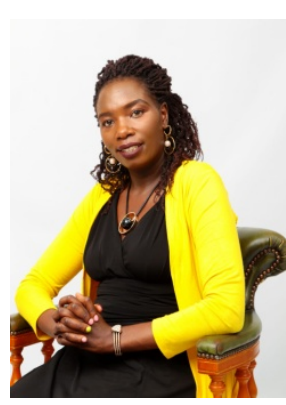

Dr. Wothaya was born in Kenya on $21^{\text {st }}$ September, 1978. She currently resides in Kenya. She graduated with a Bachelor of Science in Nursing degree at Kenya Methodist University, Meru, Kenya in 2014. She was further awarded with a Master's in Project Planning and Management degree at the University of Nairobi Kenya in 2016. She obtained a Doctor of Philosophy in Project Planning and Management degree at the University of Nairobi in 2020.

She currently works as a Program Officer in charge of Management. Organization: Family Health International (FHI360) Kenya. The program is labelled as USAID Afya Uzazi Nakuru/Baringo and is located at Nakuru County, Kenya. From April 2011 to Sept 2017, she worked at Jhpiego Kenya as the Team Lead/Technical Officer in the Reproductive Maternal neonatal Child and Adolescence Health Program. The program is labeled as USAID APHIA PLUS KAMILI and is Located in Kirinyaga and Kitui Counties, Kenya. From May 2004 to March 2011, she worked at the Ministry of Health Kenya as the provincial PMTCT Coordinator in Central Province, Kenya.

Dr. Wothaya is registered with the Nursing Council of Kenya. She was awarded with the 2008 Regional (Central Province) Nurse of the year $4^{\text {th }}$ runners up in the Nation. 